\title{
Clinical Outcomes and Quality of Vision Associated with Bilateral Implantation of a Wavefront Shaping Presbyopia Correcting Intraocular Lens
}

\author{
Kjell Gunnar Gundersen (iD) \\ Richard Potvin (iD) ${ }^{2}$ \\ 'IFocus Øyeklinikk AS, Haugesund, \\ Norway; ${ }^{2}$ Science in Vision, Bend, \\ OR, USA
}

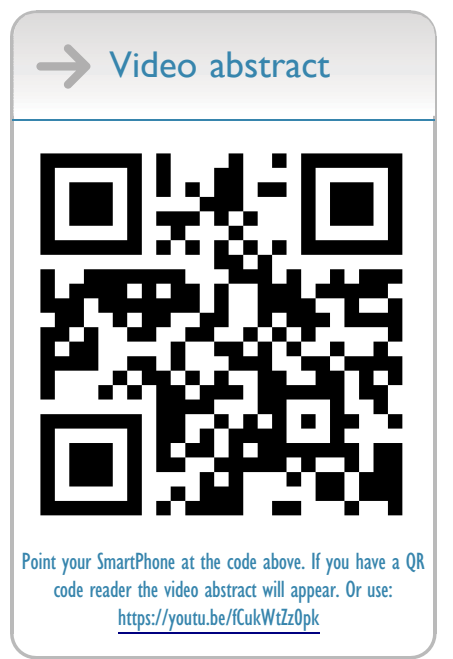

Correspondence: Kjell Gunnar Gundersen

IFocus Øyeklinikk AS, Sørhauggata III, Haugesund, 5527, Norway

Tel +47808900

Email kg@ifocus.no
Purpose: To provide normative visual acuity and quality of vision data related to bilateral implantation of a wavefront shaping presbyopia correcting intraocular lens (IOL).

Patients and Methods: This was a non-interventional research study of the refraction, visual acuity (VA) and quality of vision achieved after bilateral implantation of a wavefront shaping presbyopia correcting intraocular lens between 3 months and 12 months postsurgery. The manifest refraction, and uncorrected and distance corrected VA at near, intermediate and distance $(40 \mathrm{~cm}, 50 \mathrm{~cm}, 66 \mathrm{~cm}, 4 \mathrm{~m})$ were tested. Binocular mesopic VA at $4 \mathrm{~m}$ and uncorrected photopic low contrast $(25 \%)$ VA at $4 \mathrm{~m}$ were also tested, the latter with and without a glare source. A patient reported outcome questionnaire was administered. Defocus curve testing with and without simulated myopia in the non-dominant eye was also tested (reported elsewhere).

Results: Forty subjects completed the study. There was no statistically significant difference between the uncorrected and distance corrected VA at any distance. Mean logMAR binocular VAs were $(-0.07 \pm 0.07)$ at $4 \mathrm{~m},(0.00 \pm 0.07)$ at $66 \mathrm{~cm}$ and $(0.07 \pm 0.11)$ at $40 \mathrm{~cm}$. Uncorrected photopic low contrast VA was statistically significantly better without glare $(0.09 \pm 0.10)$ compared to with glare $(0.44 \pm 0.21, \mathrm{p}<0.01)$. Reported glare, halos and starbursts were "not at all" Or "a little" bothersome for more than $95 \%$ of subjects. Hazy vision and blurred vision were reported most often.

Conclusion: The Vivity IOL provided patients with good distance and intermediate vision, and functional near vision with low reported bother from glare, halos, or starbursts.

Keywords: non-diffractive, extended depth of focus, extended vision, cataract, presbyopia, quality of vision, wavefront shaping

\section{Plain Language Summary}

Many patients who have cataract surgery are interested in being less dependent on their glasses afterwards. Some intraocular lenses (multifocal IOLs) create several distinct focal points for far, intermediate and/or near viewing, to provide better vision at these working distances. Other lenses can extend a single focal point to help increase the range of vision. There are limits to this technology, so distance vision and computer work are generally good but near vision may not be as good as can be obtained with a multifocal IOL. However, the level of visual disturbances may be relatively lower with the extended focus IOL. The current study was designed to evaluate the clinical results obtained for patients who had a new extended range of vision IOL implanted in both eyes. The study showed that this IOL provided very good distance and intermediate vision and good near vision. Visual disturbances such as hazy vision and blurred vision were the most common reported, with low reports of glare, halos, or starbursts. 


\section{Introduction}

With modern intraocular lens (IOL) power calculation formulas, cataract surgery is justifiably considered a refractive procedure, with a high likelihood that patients will not need spectacles for distance vision when a monofocal IOL is implanted. For subjects interested in reducing their dependence on spectacles for intermediate (computer) or near (reading) vision, multifocal or extended depth of focus (EDOF) IOLs may be considered. It has been demonstrated that trifocal IOLs are most likely to provide spectacle independence at all distances. ${ }^{1-3}$ Limitations in how far the depth of focus can be extended prevent EDOF lenses from providing the same level of near vision as can be achieved with a multifocal, but they generally provide good vision at distance and intermediate. A purported advantage of EDOF IOLs is the expectation that visual disturbances will be lower, as light is not "split" to provide several distinct foci. ${ }^{3}$ However, some studies have shown that visual disturbances with a diffractive EDOF lens (Symfony, ${ }^{\circledR}$ Johnson \& Johnson Vision, Santa Ana, CA) are not significantly reduced relative to a trifocal IOL. ${ }^{4-6}$ It is recognized, though, that patients implanted with monofocal IOLs tend to have a lower incidence and severity of visual disturbances, such as glare and halos, relative to either multifocal or traditional EDOF lenses. $^{4,5}$

There are several different approaches that have been used to create EDOF lenses. ${ }^{7}$ One involves manipulating spherical aberration (SA), with one IOL designed with alternating annular zones of positive and negative SA in the IOL to increase the depth of focus. ${ }^{8,9}$ Challenges with this approach are the variability in the existing SA in each eye (making it more difficult to obtain a predictable effect) and the limits to which SA can be modified before the quality of vision degrades. Another is the use of diffractive elements to produce the EDOF effect, but as noted above the advantages of such an IOL relative to a diffractive multifocal have been questioned. A third involves the use of a limiting aperture to extend the depth of focus, a method commonly used in photography. ${ }^{10}$ The challenge with this method is that the amount of light entering the eye may be significantly reduced; as a result, this approach typically involves only monocular implantation.

The AcrySof ${ }^{\circledR}$ IQ Vivity ${ }^{\mathrm{TM}}$ IOL is based on a new optical design principle. It does not incorporate diffractive elements, nor does it target SA changes or aperture limiting technology to achieve a better range of vision. This novel presbyopia correcting IOL from Alcon (Fort Worth, TX) incorporates proprietary wavefront shaping (X-WAVETM) technology to produce an extended focal length that channels almost all the incoming light through the IOL in that specific range. ${ }^{11}$ The low amount of "unused" light is expected to reduce the potential for visual disturbances, because out-of-focus light is generally the root cause of disturbances such as halos and glare. In several clinical studies the reported visual disturbances with this IOL were not different from those observed with a monofocal IOL, but intermediate and near vision were better than can be achieved with a monofocal IOL. ${ }^{12,13}$ The lens is a 1-piece, hydrophobic aspheric posterior chamber IOL with ultraviolet protection and a blue-light filter. It has a $6 \mathrm{~mm}$ optical zone with a modified central $2.2 \mathrm{~mm}$ region to create the "stretched" wavefront.

The purpose of the current study was to provide objective and subjective normative clinical outcomes data for patients who have the Vivity IOL bilaterally implanted.

\section{Patients and Methods}

This study was a non-interventional single-arm study of visual outcomes and quality of vision after successful bilateral implantation of the Vivity IOL. A regional ethics committee (REK, Norway) approved the study, and enrolled subjects signed an appropriate informed consent document. As a non-interventional study, there was no clinical trial registration requirement. The study was conducted in accordance with good clinical practice (GCP) and the tenets of the Declaration of Helsinki were observed. Data are not available for sharing.

Eligible patients had to have had previous uncomplicated bilateral cataract surgery with binocular implantation of the Vivity lens more than 3 months but less than a year prior to their enrollment. They had to have a best-corrected monocular and binocular distance visual acuity (VA) of 20/40 (0.3 $\log$ MAR $)$ or better. Subjects with previous corneal surgery, ocular pathology, or significant posterior capsular opacity (PCO) were excluded. One surgeon (KGG) had performed all surgeries. Eyes with both toric and non-toric versions of the Vivity IOL were included. To be enrolled, the inclusion and exclusion criteria had to be met in both eyes of any potential subject.

Potential participants were identified from the clinical records of the site and screened to establish their eligibility, based on the inclusion and exclusion criteria above. If 
eligible, they were asked to participate in two postoperative diagnostic visits (one to three weeks apart); this was to reduce potential fatigue related to testing multiple defocus curves. Clinical evaluations included a manifest refraction, uncorrected and distance corrected VA at near $(40 \mathrm{~cm}$ and $50 \mathrm{~cm})$ intermediate $(66 \mathrm{~cm})$, and distance $(4 \mathrm{~m})$ Uncorrected photopic low contrast (25\%) VA at $4 \mathrm{~m}$ was also tested, with and without a glare source. VA data were collected using the M\&S Technologies Clinical Trial Suite (Niles, IL, USA). A patient reported outcome questionnaire related to quality of vision was also administered (the Quality of Vision questionnaire, or Q of V). ${ }^{14}$ This Rasch-scored questionnaire measures the reported Frequency, Severity and Degree of Bother associated with 10 distinct visual disturbances, such as glare, halos, starbursts, and blurred vision. In addition to the above, four binocular defocus curves were collected for each subject based on different levels of simulated monovision. Defocus curve results are reported in a separate publication. $^{15}$

Demographic and surgical planning data were obtained from the subjects' clinical records. Surgeries were performed bilaterally on the same day, with one eye targeted for the least-plus sphere from the surgery planning results and the contralateral eye targeted for the least-minus sphere. Toric planning was based on software that included consideration of posterior corneal astigmatism. One optometrist conducted all diagnostic visits. Detailed statistical analyses were performed using STATISTICA, version 12 (TIBCO Software Inc., Palo Alto, CA, USA).

\section{Results}

Forty subjects were recruited for the study and completed the two diagnostic visits. A summary of the relevant demographic, preoperative and postoperative data is contained in Table 1. No subject had any adverse events identified at either diagnostic visit. Fifteen percent (12/

Table I Subject $(n=40)$ Demographics and Refractive Data $(80$ Eyes)

\begin{tabular}{|l|c|}
\hline Male/Female & $\mathbf{2 5 / 1 5}$ \\
\hline Age (years) & $59 \pm 8(48$ to $8 I)$ \\
Non-toric/Toric & $68 / 12$ \\
Days post-surgery & $186 \pm 98(9 \mathrm{I}$ to $4 \mathrm{I} 3)$ \\
MRSE (D) & $-0.06 \pm 0.36(-1.00$ to 1.12$)$ \\
Cylinder (D) & $-0.37 \pm 0.29(-1.25$ to 0.00$)$ \\
\hline
\end{tabular}

Abbreviations: MRSE, mean refraction spherical equivalent; $D$, diopter.
80) of eyes had a toric IOL implanted. There was no statistically significant difference in either the postoperative spherical equivalent refraction $(p=0.51)$ or the refractive cylinder $(\mathrm{p}=0.57)$ between the non-toric and toric eyes. Overall, $90 \%$ of eyes had a spherical equivalent refraction within $0.50 \mathrm{D}$ of plano and $83 \%$ had $\leq 0.50$ $\mathrm{D}$ of refractive cylinder. Only one eye was more than $0.50 \mathrm{D}$ hyperopic. Three quarters of all eyes $(60 / 80)$ had a residual spherical equivalent refraction within $0.50 \mathrm{D}$ of plano with $\leq 0.50 \mathrm{D}$ of refractive cylinder. Figure 1 shows the distribution of residual refractive error. YAG capsulotomies were performed in $9 \%(7 / 80)$ of eyes, all prior to their study visit.

Figure 2 shows the uncorrected and distance corrected binocular visual acuity at all test distances. There was no statistically significant difference between the uncorrected and distance corrected VA at any distance. A repeated measures ANOVA showed that the VA at the different test distances was statistically significantly different but post-hoc testing showed there was no statistically significant difference between $66 \mathrm{~cm}$ and $50 \mathrm{~cm}$ for either the uncorrected $(\mathrm{p}=0.6)$ or distance corrected $(\mathrm{p}=0.9)$ condition.

The binocular mesopic uncorrected and distance corrected near VA at $40 \mathrm{~cm}$ and $50 \mathrm{~cm}$ was measured. There was no statistically significant difference between the uncorrected and corrected states at either distance. The mean binocular mesopic uncorrected VA was about $0.1 \pm$ 0.10 in both cases at both distances.

Figure 3 shows the distribution of the uncorrected binocular photopic low contrast VA with and without glare at $4 \mathrm{~m}$. There was a statistically significant difference between the no glare and glare conditions $(0.09 \pm 0.10$ with no glare vs $0.44 \pm 0.21$ with glare, $\mathrm{p}<0.01$ ). The low contrast acuity in no glare conditions was about 2 lines worse than the high contrast BDVA reported above.

Figure 4 shows a box-whisker plot of the distributions of the Rasch-scored results of the Quality of Vision questionnaire, showing summary data for the Frequency, Severity and Degree of Bother of visual disturbances with this IOL. There was no correlation between the $\mathrm{Q}$ of $\mathrm{V}$ scores and the follow-up time. There was also no apparent correlation between the Q of V scores and the refractive status or visual acuity results. Figure 5 shows the average responses related to each of the specific visual disturbances included in the questionnaire. For glare, halos and starbursts, three of the visual disturbances most often reported with multifocal and EDOF IOLs, 93\% or more of 


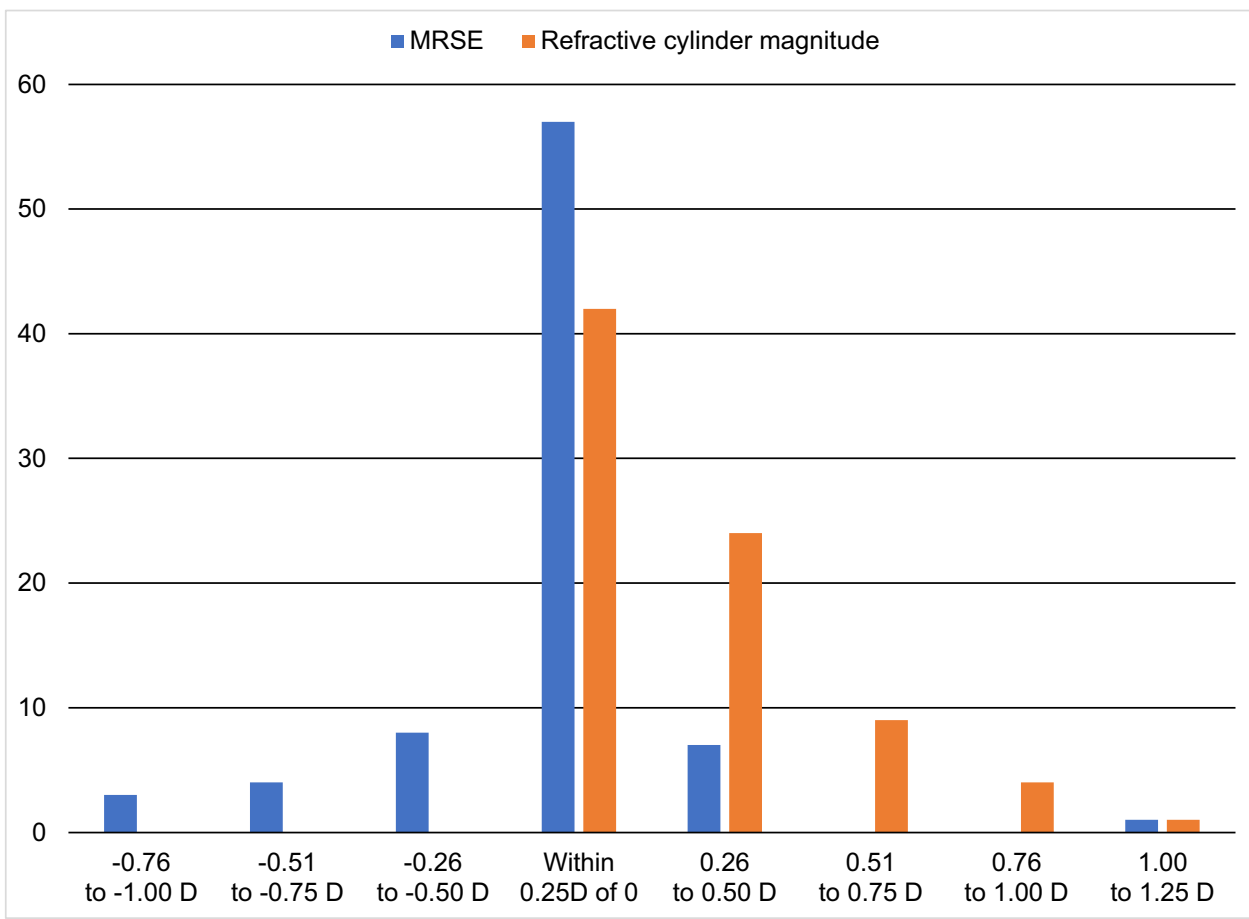

Figure I Postoperative MRSE and refractive cylinder magnitude.

Abbreviations: MRSE, mean refraction spherical equivalent; D, diopter.

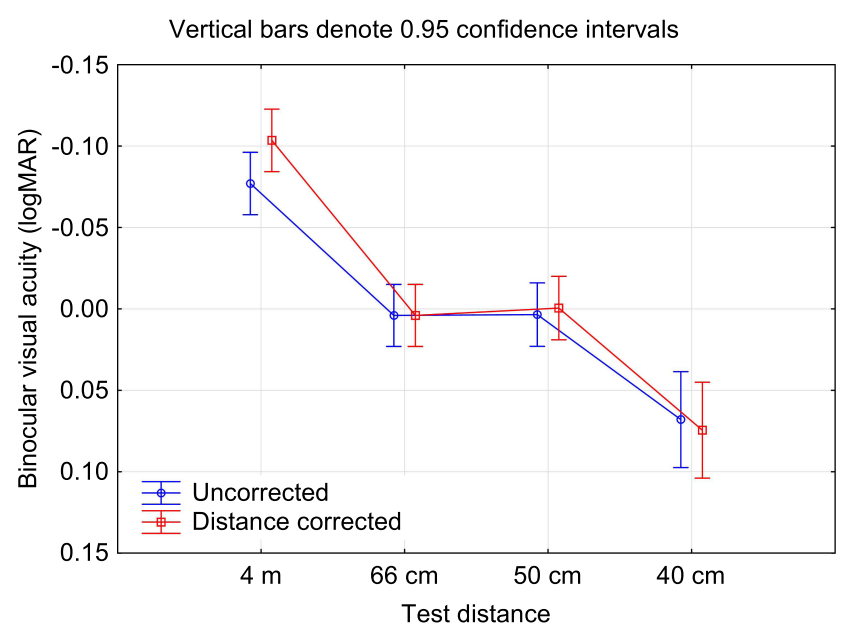

Figure 2 Uncorrected and distance corrected binocular visual acuity at various test distances.

Abbreviation: logMAR, log of the minimum angle of resolution.

subjects reported a frequency of "never" or "occasionally", $90 \%$ a severity of "not at all" or "mild" and 95\% a degree of bother of "not at all" or "a little". The most frequently reported visual disturbances in the current study were hazy vision, blurred vision and focusing difficulties.

\section{Discussion}

These results are among the first reported for this IOL in a clinical setting. As noted earlier, defocus curve results are reported in a separate publication. The distance corrected and uncorrected VA results at all tested distances were slightly better than those reported in the Vivity FDA Summary of Safety and Effectiveness document. ${ }^{12}$ They were also slightly better than the results reported by Bala et $\mathrm{al}^{13}$ and Arrigo. ${ }^{16}$

The Vivity lens also seemed to provide intermediate VA results that were better than those reported for several other EDOF lenses, ${ }^{17,18}$ and a monofocal IOL enhanced to improve intermediate vision. ${ }^{19}$ The combined use of a small aperture IOL (IC-8, Acufocus) and the Symfony diffractive EDOF lens appeared to provide similar intermediate vision to that obtained with the Vivity lens in the current study, ${ }^{20}$ as did one other EDOF lens (Mini Well ${ }^{\circledR}, \mathrm{SiFi}$ Medtec, Catania, Italy). ${ }^{9}$ In all the studies mentioned above the binocular distance vision appeared comparable, though the mean binocular near vision measured in the current study (0.07 logMAR) was almost 1 line to more than two lines better than results from the different EDOF IOLs in these other studies. As always, comparisons between different clinical studies must be evaluated with due consideration for different testing conditions, test distances (eg, intermediate), inclusion/exclusion criteria and follow-up time. 


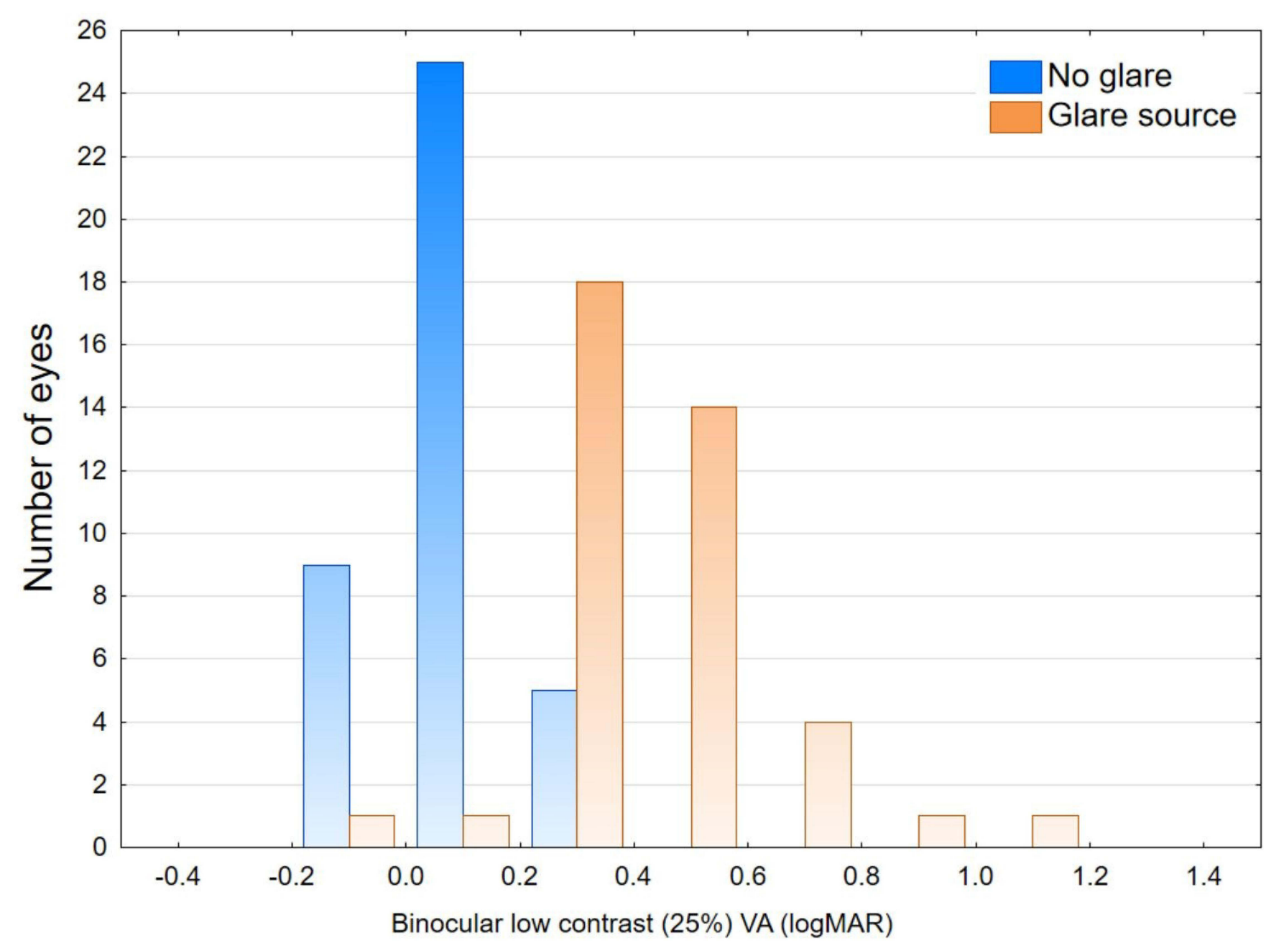

Figure 3 Uncorrected photopic low contrast binocular visual acuity at $4 \mathrm{~m}$.

Abbreviation: $\log M A R, \log$ of the minimum angle of resolution.

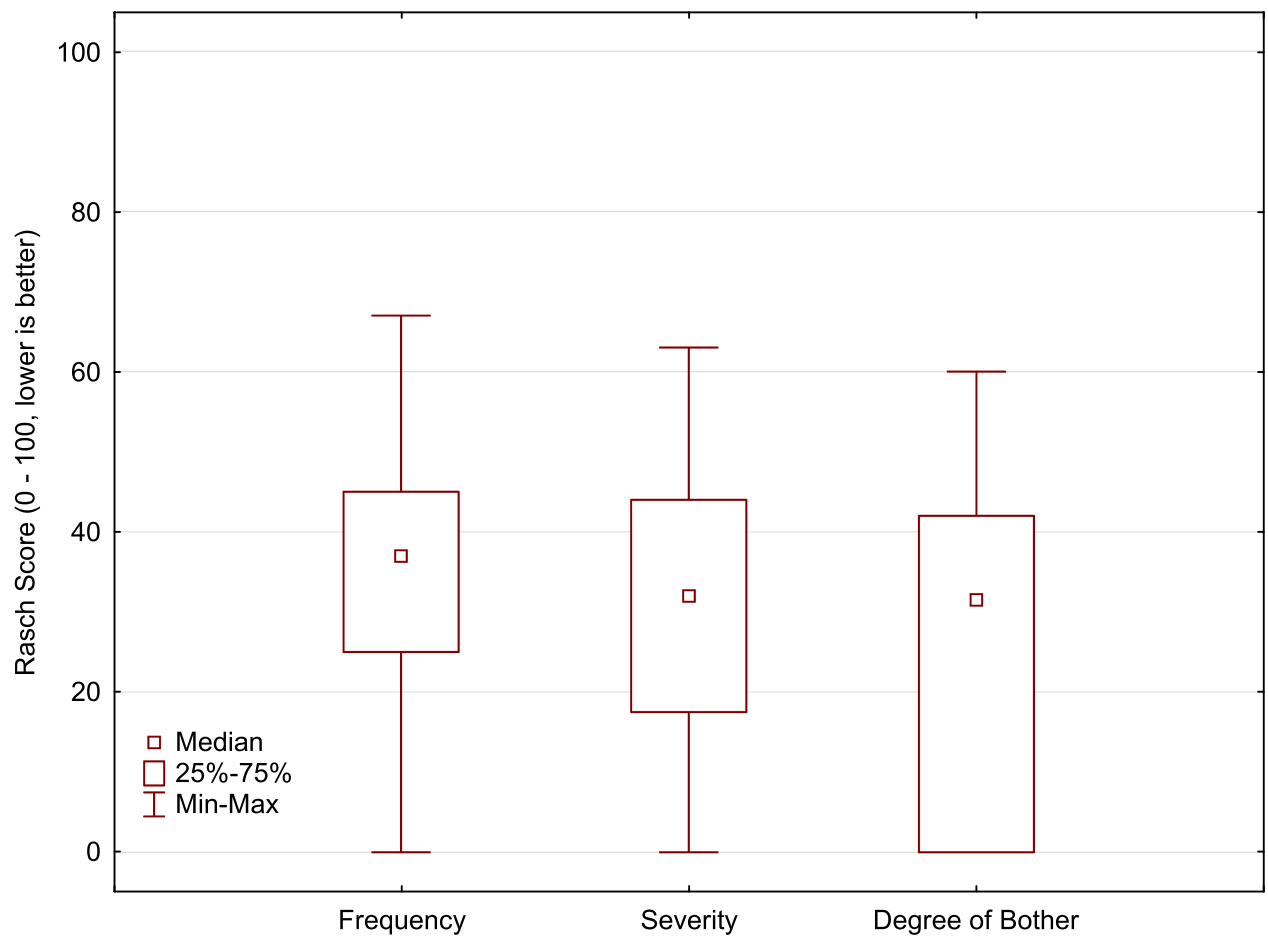

Figure 4 Distribution of aggregate results for the Quality of Vision questionnaire.

Mesopic VA at $50 \mathrm{~cm}$ was similar to the photopic VA measured at the same distance and was about a line better than mesopic VA at $40 \mathrm{~cm}$. No specific luminance was recorded for these measures, so it is possible the level of room illumination was not dimmed sufficiently to result in a measurable difference. 


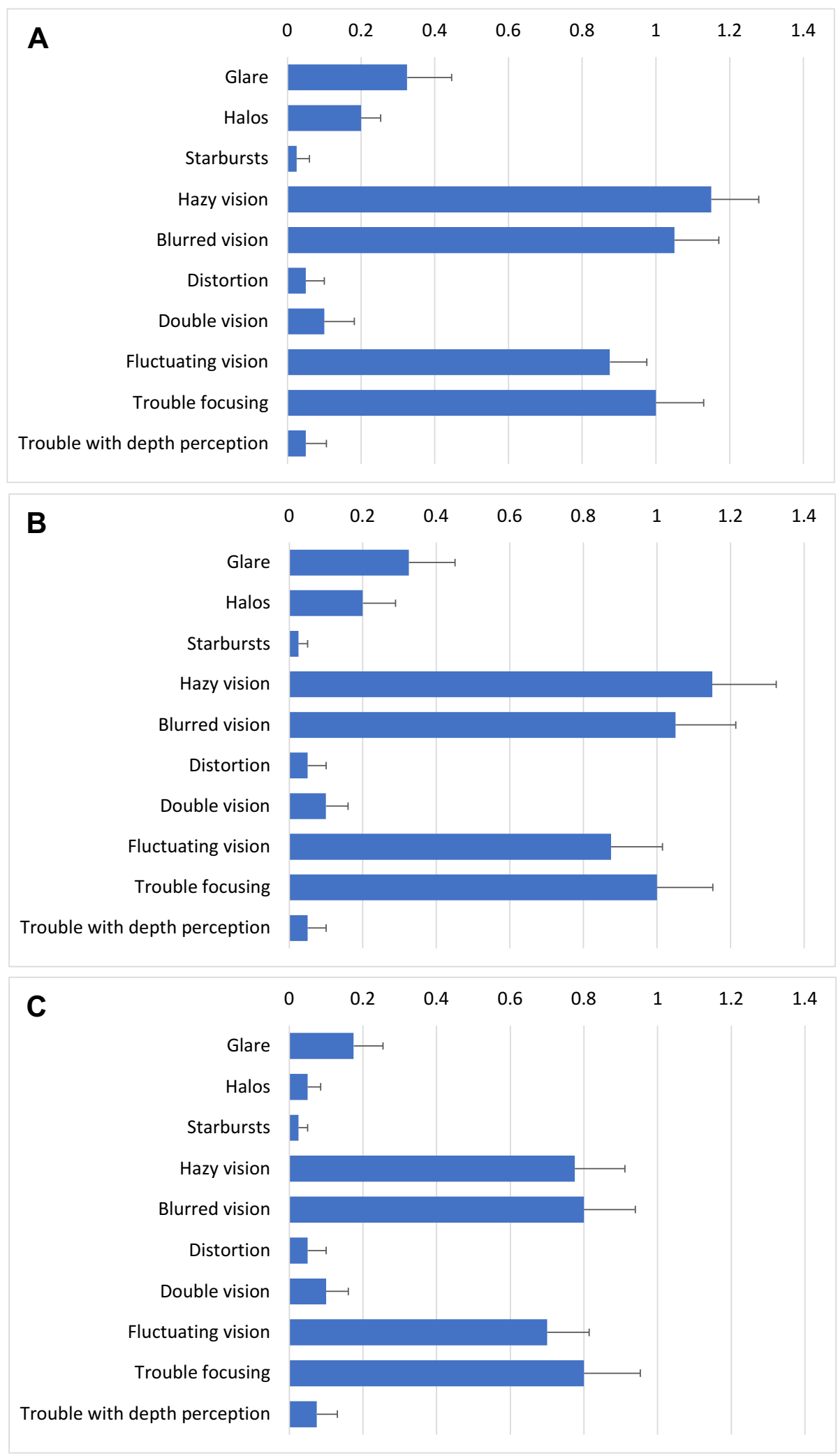

Figure 5 Detailed Quality of Vision questionnaire responses, with scoring on a 0-3 ordinal scale (lower is better). (A) Frequency, (B) severity, (C) degree of bother.

Low contrast visual acuity at distance was reduced by about 2 lines in the no glare condition and about 5 lines with added glare. These results were slightly better than recorded for an EDOF lens and a trifocal IOL at the same practice, though the testing methodology was slightly different for both studies. ${ }^{21}$ 
The overall Quality of Vision scores were somewhat higher than expected, though visual disturbances such as glare, halos and starbursts were reported infrequently and were classified as of little or no bother. The frequency, severity and degree of bother related to glare, halos and starbursts reported here are lower than those reported by Bala et $\mathrm{al}^{13}$ and Arrigo et $\mathrm{a}^{15}$ for the same lens using the same questionnaire; subjects were evaluated at 3 months postoperative in these two studies. ${ }^{13}$ In the current study, hazy vision and blurred vision were reported most often. In contrast to this, raw scores from Arrigo et al indicated that blurred vision and trouble focusing were of most concern to subjects (after glare and halos), but hazy vision and fluctuating vision were never, or only rarely, reported. While a different questionnaire was used for the Vivity trial to obtain FDA approval, the questions were very similar; the level of hazy vision reported here appears higher than was reported in that previous trial. ${ }^{12} \mathrm{It}$ is unclear whether the questionnaire results obtained in the current study were related to patient expectations, patient instructions related to the questionnaire or regional differences in responses; the latter issue has been noted in the past. ${ }^{22}$ Residual refractive error is also a possible contributing factor, though we found no correlation. Ocular surface disease may be another contributing factor. Further studies would be helpful in determining if the results at this site were anomalous. Aggregate raw scores appeared lower (better) than those previously reported for an EDOF lens and bifocal lens using the same questionnaire. ${ }^{23}$

There are limitations to the current study. It was at a single center and the number of subjects was limited. Subjects were recruited from patients in the practice who had already been binocularly implanted with the Vivity IOL, so there was a relatively wide range of follow-up. Mesopic vision illumination levels were not recorded. The range of postoperative follow-up was also relatively high (91 to 413 days), which might be a concern related to different levels of neuroadaptation. However, we found no correlation between quality of vision scores and follow-up time; recent research has suggested that neuroadaptation occurs within 3 months of implantation of either a monofocal or multifocal IOL. ${ }^{24}$

In conclusion, the Vivity IOL provided patients with good distance and intermediate vision, and functional near vision; with most subjects reporting little or no bother from glare, halos, or starbursts.

\section{Acknowledgments}

Steffen Østenstad, MSc, of IFocus Øyeklinikk AS assisted with diagnostic testing and data collection/checking. This work was supported by as an investigator-initiated study grant funded by Alcon (IIT\#61478839). Preliminary results from this study were presented at the 2021 American Society of Cataract and Refractive Surgery conference (Las Vegas, USA, July 23-27, 2021) and the 2021 European Society of Cataract and Refractive Surgery (Amsterdam, NL, October 8-11, 2021) in conference lectures.

\section{Disclosure}

Drs. Gundersen and Potvin are consultants to Alcon. Dr Potvin reports personal fees from Alcon and Carl Zeiss Meditec, outside the submitted work. The authors report no other conflicts of interest in this work.

\section{References}

1. Rodov L, Reitblat O, Levy A, Assia EI, Kleinmann G. Visual outcomes and patient satisfaction for trifocal, extended depth of focus and monofocal intraocular lenses. J Refract Surg. 2019;35 (7):434-440. doi:10.3928/1081597X-20190618-01

2. Pedrotti E, Carones F, Talli P, et al. Comparative analysis of objective and subjective outcomes of two different intraocular lenses: trifocal and extended range of vision. BMJ Open Ophthalmol. 2020;5: e000497. doi:10.1136/bmjophth-2020-000497

3. Hovanesian JA, Jones M, Allen Q. The PanOptix trifocal IOL vs the ReSTOR 2.5 active focus and ReSTOR 3.0-add multifocal lenses: a study of patient satisfaction, visual disturbances, and uncorrected visual performance. Clin Ophthalmol. 2021;15:983-990. doi:10.2147/OPTH. S285628

4. Monaco G, Gari M, Di Censo F, Poscia A, Ruggi G, Scialdone A. Visual performance after bilateral implantation of 2 new presbyopia-correcting intraocular lenses: trifocal versus extended range of vision. J Cataract Refract Surg. 2017;43(6):737-747. doi:10.1016/j.jcrs.2017.03.037

5. Cochener B, Boutillier G, Lamard M, Auberger-Zagnoli C. A comparative evaluation of a new generation of diffractive trifocal and extended depth of focus intraocular lenses. J Refract Surg. 2018;34(8):507-514. doi:10.3928/1081597X-20180530-02

6. Singh B, Sharma S, Dadia S, Bharti N, Bharti S. comparative evaluation of visual outcomes after bilateral implantation of a diffractive trifocal intraocular lens and an extended depth of focus intraocular lens, eye \& contact lens. Sci Clin Pract. 2020;46(5):314-318. doi:10.1097/ICL.0000000000000637

7. Kohnen T, Suryakumar R. Extended depth-of-focus technology in intraocular lenses. J Cataract Refract Surg. 2020;46(2):298-304. doi:10.1097/j.jcrs.0000000000000109

8. Ruiz-Mesa R, Blanch-Ruiz J, Ruiz-Santos M, Montés-Micó R. Optical and visual quality assessment of an extended depth-of-focus intraocular lens based on spherical aberration of different sign. Int Ophthalmol. 2021;41(3):1019-1032. doi:10.1007/s10792-020-01659-z

9. Savini G, Balducci N, Carbonara C, et al. Functional assessment of a new extended depth-of-focus intraocular lens. Eye (Lond). 2019;33 (3):404-410. doi:10.1038/s41433-018-0221-1

10. Hooshmand J, Allen P, Huynh T, et al. Small aperture IC-8 intraocular lens in cataract patients: achieving extended depth of focus through small aperture optics. Eye (Lond). 2019;33(7):1096-1103. doi:10.1038/s41433-019-0363-9

11. Kohnen T. Nondiffractive wavefront-shaping extended range-ofvision intraocular lens. J Cataract Refract Surg. 2020;46 (9):1312-1313. doi:10.1097/j.jcrs.0000000000000247 
12. US FDA. AcrySof ${ }^{\mathrm{TM}} \mathrm{IQ}$ Vivity $^{\mathrm{TM}}$ extended vision intraocular lens (IOL): summary of safety and effectiveness data. Available from: https://www.accessdata.fda.gov/cdrh_docs/pdf/P930014S126B.pdf. Accessed March 7, 2021.

13. Bala C, Poyales F, Guarro M, et al. Multi-country clinical outcomes of a new nondiffractive presbyopia-correcting intraocular lens. J Cataract Refract Surg. 2021;Publish Ahead of Print. doi:10.1097/ j.jcrs.0000000000000712.

14. McAlinden C, Pesudovs K, Moore JE. The development of an instrument to measure quality of vision: the Quality of Vision (QoV) questionnaire. Invest Ophthalmol Vis Sci. 2010;51(11):5537-5545. doi:10.1167/iovs.10-5341

15. Gundersen KG, Potvin R. The effect of spectacle-induced low myopia in the non-dominant eye on the binocular defocus curve with a non-diffractive extended vision intraocular lens. Clin Ophthalmol. 2021;15:3541-3547. doi:10.2147/OPTH.S329922

16. Arrigo A, Gambaro G, Fasce F, Aragona E, Figini I, Bandello F. Extended depth-of-focus (EDOF) AcrySof ${ }^{\circledR}$ IQ Vivity ${ }^{\circledR}$ intraocular lens implant: a real-life experience. Graefes Arch Clin Exp Ophthalmol. 2021;259:2717-2722. doi:10.1007/s00417-021-05245-6

17. Iradier MT, Cruz V, Gentile N, Cedano P, Piñero DP. Clinical outcomes with a novel extended depth of focus presbyopia-correcting intraocular lens: pilot study. Clin Ophthalmol. 2021;15:1215-1221. doi:10.2147/OPTH.S297985

18. Reinhard T, Maier P, Böhringer D, et al. Comparison of two extended depth of focus intraocular lenses with a monofocal lens: a multi-centre randomised trial. Graefes Arch Clin Exp Ophthalmol. 2021;259(2):431-442. doi:10.1007/s00417-020-04868-5
19. Mencucci R, Cennamo M, Venturi D, Vignapiano R, Favuzza E. Visual outcome, optical quality, and patient satisfaction with a new monofocal IOL, enhanced for intermediate vision: preliminary results. J Cataract Refract Surg. 2020;46(3):378-387. doi:10.1097/j. jcrs.0000000000000061

20. Schojai M, Schultz T, Jerke C, Böcker J, Dick HB. Visual performance comparison of 2 extended depth-of-focus intraocular lenses. $J$ Cataract Refract Surg. 2020;46(3):388-393. doi:10.1097/j. jcrs.0000000000000068

21. Gundersen KG, Potvin R. Comparing visual acuity, low contrast acuity and contrast sensitivity after trifocal toric and extended depth of focus toric intraocular lens implantation. Clin Ophthalmol. 2020;14:1071-1078. doi:10.2147/OPTH.S253250

22. Tran DB, Owyang A, Hwang J, Potvin R. Visual acuity, quality of vision, and patient-reported outcomes after bilateral implantation with a trifocal or extended depth of focus intraocular lens. Clin Ophthalmol. 2021;15:403-412. doi:10.2147/OPTH. $\mathrm{S} 295503$

23. Liu X, Song X, Wang W, et al. Comparison of the clinical outcomes between echelette extended range of vision and diffractive bifocal intraocular lenses. J Ophthalmol. 2019;2019:5815040. doi:10.1155/ 2019/5815040

24. Zhang L, Lin D, Wang Y, et al. Comparison of visual neuroadaptations after multifocal and monofocal intraocular lens implantation. Front Neurosci. 2021;15:648863. doi:10.3389/fnins. 2021.648863
Clinical Ophthalmology

\section{Publish your work in this journal}

Clinical Ophthalmology is an international, peer-reviewed journal covering all subspecialties within ophthalmology. Key topics include: Optometry; Visual science; Pharmacology and drug therapy in eye diseases; Basic Sciences; Primary and Secondary eye care; Patient Safety and Quality of Care Improvements. This journal is indexed on PubMed

\section{Dovepress}

Central and CAS, and is the official journal of The Society of Clinical Ophthalmology (SCO). The manuscript management system is completely online and includes a very quick and fair peer-review system, which is all easy to use. Visit http://www.dovepress.com/ testimonials.php to read real quotes from published authors. 